\title{
DISTRIBUCIÓN ESPACIAL DEL AMACHAMIENTO DEL FRIJOL (Aphelenchoides besseyi CHRISTIE) EN CAMPO ${ }^{1}$
}

\author{
Néstor Felipe Chaves-Barrantes ${ }^{2}$, Carlos Manuel Araya-Fernández ${ }^{3}$
}

\section{RESUMEN}

Distribución espacial del amachamiento del frijol (Aphelenchoides besseyi Christie) en campo. El objetivo del presente trabajo fue determinar la distribución espacial del amachamiento en campo. La investigación se realizó entre mayo y julio del 2009 en las comunidades de Veracruz de Pérez Zeledón, San José y Concepción de Buenos Aires, Puntarenas, Costa Rica. Para ello se evaluó la incidencia de la enfermedad por punto de siembra en dos parcelas de frijol provenientes de distintas rotaciones (arroz/frijol y maíz/frijol), con lo cual, posteriormente se determinó la distribución espacial de la enfermedad en campo por medio de un análisis de corridas y los índices de varianza/media (V/M), Lloyd y Morisita. En el análisis de corridas se obtuvo un valor promedio de $\mathrm{Z}$ de $-1,06$ para la parcela sembrada previamente con arroz, y de $-0,98$ para la parcela sembrada previamente con maíz, lo que indica una tendencia a la agregación. Los índices de V/M, Lloyd y Morisita fueron mayores que 1 en ambas parcelas, por lo que se determinó que el amachamiento presentó un patrón espacial agregado (en parches) en condiciones de campo. El patrón espacial del amachamiento en campo tiende a enmascararse debido a la rotación con cultivos hospederos de Aphelenchoides besseyi, - su agente causal -, en especial la rotación maíz/frijol donde el productor redistribuye el inóculo de la enfermedad cuando esparce las cañas de maíz sobre el terreno de cultivo.

\begin{abstract}
Spatial distribution pattern of common bean "amachamiento" (Aphelenchoides besseyi Christie) in field. The objective of the research was to determine the spatial distribution pattern of common bean "amachamiento" in field. It was carried out from May through July 2009 in Pérez Zeledón, San José Province, and Buenos Aires, Puntarenas Province, in Costa Rica. Disease incidence in micro plots was evaluated in two commercial fields following two different crop rotation systems: rice/common bean and corn/ common bean. Spatial distribution of "amachamiento" in field was determined by using the ordinary runs analysis, variance to mean ratio (V/M), Lloyd and Morisita's index. The ordinary runs analysis showed $-1.06 \mathrm{Z}$ value for the first crop rotation system and $-0,98$ for the second one, what suggested an aggregated spatial pattern (patches) of the disease in the field. The M/V, Lloyd and Morisita's index were over 1 in both fields, what confirmed the aggregated pattern. Patches of "amachamiento" in the field can be masked by crop rotation with hosts of the nematode Aphelenchoides besseyi - its causal agent -, especially corn/common bean rotation since the farmer spread over the field the surviving inoculum in crop debris before the common bean crop.
\end{abstract}

Keywords: false angular leaf spot, foliar nematode, spatial pattern, Phaseolus vulgaris L.

Palabras clave: falsa mancha angular, nematodo foliar, patrón espacial, Phaseolus vulgaris L.

\footnotetext{
Recibido: 3 de octubre, 2013. Aceptado: 18 de marzo, 2014. Parte de la tesis del primer autor para obtener el título y grado de Maestría Académica en Ciencias Agrícolas y Recursos Naturales con énfasis en Protección de Cultivos, Universidad de Costa Rica. Proyecto V.I. 736-A4-141, Universidad de Costa Rica.

2 Programa de Leguminosas, Estación Experimental Agrícola Fabio Baudrit Moreno, Universidad de Costa Rica. Apdo. postal 183-4050 Alajuela, Costa Rica.nfchaves@gmail.com

3 Laboratorio de Fitopatología, Escuela de Ciencias Agrarias, Universidad Nacional. Apdo. postal 86-3000 Heredia, Costa Rica. caraya@una. ac.cr
} 


\section{INTRODUCCIÓN}

El frijol es un cultivo muy importante para pequeños productores en las regiones Brunca y Huetar Norte de Costa Rica (Salazar, 2002 y 2003), y es consumido por el $96 \%$ de la población costarricense (Rodríguez y Fernández, 2008). Los productores nacionales de frijol tienen grandes limitaciones, entre ellas los problemas fitosanitarios (Araya, 2001; Araya y Hernández, 2003), donde destaca la enfermedad conocida como amachamiento, causada por el nematodo Aphelenchoides besseyi Christie (Chaves, 2011; Chaves et al., 2013).

El amachamiento del frijol se caracteriza por ocasionar lesiones necróticas angulares en las hojas inferiores (Salas y Vargas, 1984; Barrantes, 2006) y diversos grados de deformación de la nervadura central y lámina foliar en los estratos superiores (Araya y Hernández, 2006; Chaves, 2011; Chaves et al., 2013). Además, la planta infectada presenta una reducción significativa en la formación de vainas (71-80\%) y en el rendimiento (69-85\%) (Chaves y Araya, 2012b).

El patrón de distribución de una enfermedad en el campo, permite dar una idea del tipo de problema fitopatológico que se enfrenta. Las enfermedades causadas por nematodos generalmente presentan gradientes de dispersión pronunciados y patrones espaciales típicamente agregados, debido a que la escasa movilidad de estos organismos les impide trasladarse a grandes distancias (Campbell y Noe, 1985; Noe y Campbell, 1985; Arauz, 2011). El nematodo A. besseyi presenta movilidad reducida y su diseminación se da por salpique de lluvia o por contacto entre plantas húmedas (Christie, 1982; Barrantes, 2006; Araya, 2008), por lo que las plantas enfermas siempre se encuentran próximas unas de otras.

El estudio de la distribución espacial de enfermedades es importante para el diseño de una estrategia de manejo, ya que se pueden aplicar medidas de combate en forma localizada y no generalizada (Arauz, 2011). Este aspecto es de suma importancia en el caso de los nematicidas, ya que la mayoría tienden a ser bastante tóxicos, volátiles, con poca especificidad, y altamente contaminantes para el agua del subsuelo y la capa de ozono (Chitwood, 2002). Sin embargo, en ocasiones no es fácil detectar el patrón de distribución espacial de una enfermedad en campo, por lo que se debe recurrir a herramientas analíticas cuantitativas como el análisis de corridas y los índices de varianza/media (V/M), Lloyd y Morisita (Campbell y Madden, 1990). El objetivo del presente trabajo fue determinar la distribución espacial del amachamiento en condiciones de campo.

\section{MATERIALES Y MÉTODOS}

Con el propósito de determinar el patrón espacial del amachamiento en campo, en mayo del 2009 se delimitaron dos parcelas experimentales de 25 hileras por 25 puntos de siembra (dos a tres plantas), de aproximadamente $125 \mathrm{~m}^{2}$ cada una. Una se localizó en un campo comercial de frijol previamente sembrado con maíz (rotación maíz/frijol), ubicado en Veracruz (N 0905'; O 83³2'; 657 msnm), cantón de Pérez Zeledón, provincia de San José. La otra parcela se ubicó en Concepción de Pilas (N 0906'; O 83²9'; $734 \mathrm{msnm}$ ), cantón de Buenos Aires, provincia de Puntarenas, en un campo comercial de frijol donde se tuvo arroz como cultivo anterior (rotación arroz/frijol). Ambos campos comerciales se encontraban sembrados con la variedad comercial Cabécar, de grano rojo. Se seleccionaron estas rotaciones debido a que el arroz y el maíz son cultivos hospederos de A. besseyi, son las rotaciones más comunes en la región y garantizaban un nivel apropiado de inóculo inicial para el ciclo con frijol (Chaves y Araya, 2012a). Las parcelas se delimitaron cuando el frijol se encontraba en la etapa de emergencia (V1, ocho días después de la siembra), a partir de un punto al azar en el campo comercial.

En julio 2009, cuando las plantas alcanzaron la etapa de floración (R6), se evaluó la incidencia de amachamiento en cada uno de los puntos de siembra de las parcelas experimentales establecidas. Se evaluó la enfermedad en esta etapa fenológica, porque la incidencia correlaciona mejor con las pérdidas en campo (Chaves y Araya, 2012b).

Con los datos de incidencia por punto de siembra y con la ubicación exacta de cada punto en la parcela establecida, se procedió a dibujar mapas de la distribución de la enfermedad en campo. Con los datos de puntos de siembra enfermos y su ubicación, se aplicó un análisis de corridas y se calcularon los índices de varianza/media (V/M), Lloyd y Morisita (Campbell y Madden, 1990) para determinar el arreglo espacial de la enfermedad en campo. El análisis de corridas se 
aplicó a cada hilera, debido a la limitación del mismo para aplicarse a un conjunto de hileras (Campbell y Madden, 1990). Para el cálculo de los índices de agregación se dividió cada parcela en cuadrículas de cinco hileras por cinco puntos de siembra, para un total de veinticinco, y en cada una de ellas se cuantificó el número de puntos de siembra enfermos como medida del nivel de la enfermedad. El análisis de corridas y el cálculo de los índices de agregación se realizaron mediante una hoja electrónica de Excel.

\section{RESULTADOS Y DISCUSIÓN}

El patrón espacial del amachamiento en condiciones de campo se evaluó en dos siembras comerciales de frijol, una donde previamente hubo arroz (Figura 1) y otra donde hubo maíz (Figura 2), los cultivos de rotación empleados con mayor frecuencia en la zona (Chaves y Araya, 2012a). En ambos casos se observó una tendencia de la enfermedad a presentarse en parches (Figuras 1 y 2), más definidos en la parcela de rotación con arroz que en la de rotación con maíz. Esta tendencia es debida a la reducida movilidad del nematodo $A$. besseyi (Christie, 1982) y su forma de diseminación, vía salpique o por agua libre en el follaje (Fortuner y Orton, 1975; Barrantes, 2006; Araya, 2008), donde la cercanía de las plantas y el traslape de sus doseles en campo, constituyen factores fundamentales para el patrón de distribución espacial que presenta el amachamiento en condiciones de campo.

Al realizar el análisis de corridas, la agregación del amachamiento en campo no fue tan evidente, al igual que lo observado en el patrón espacial de la

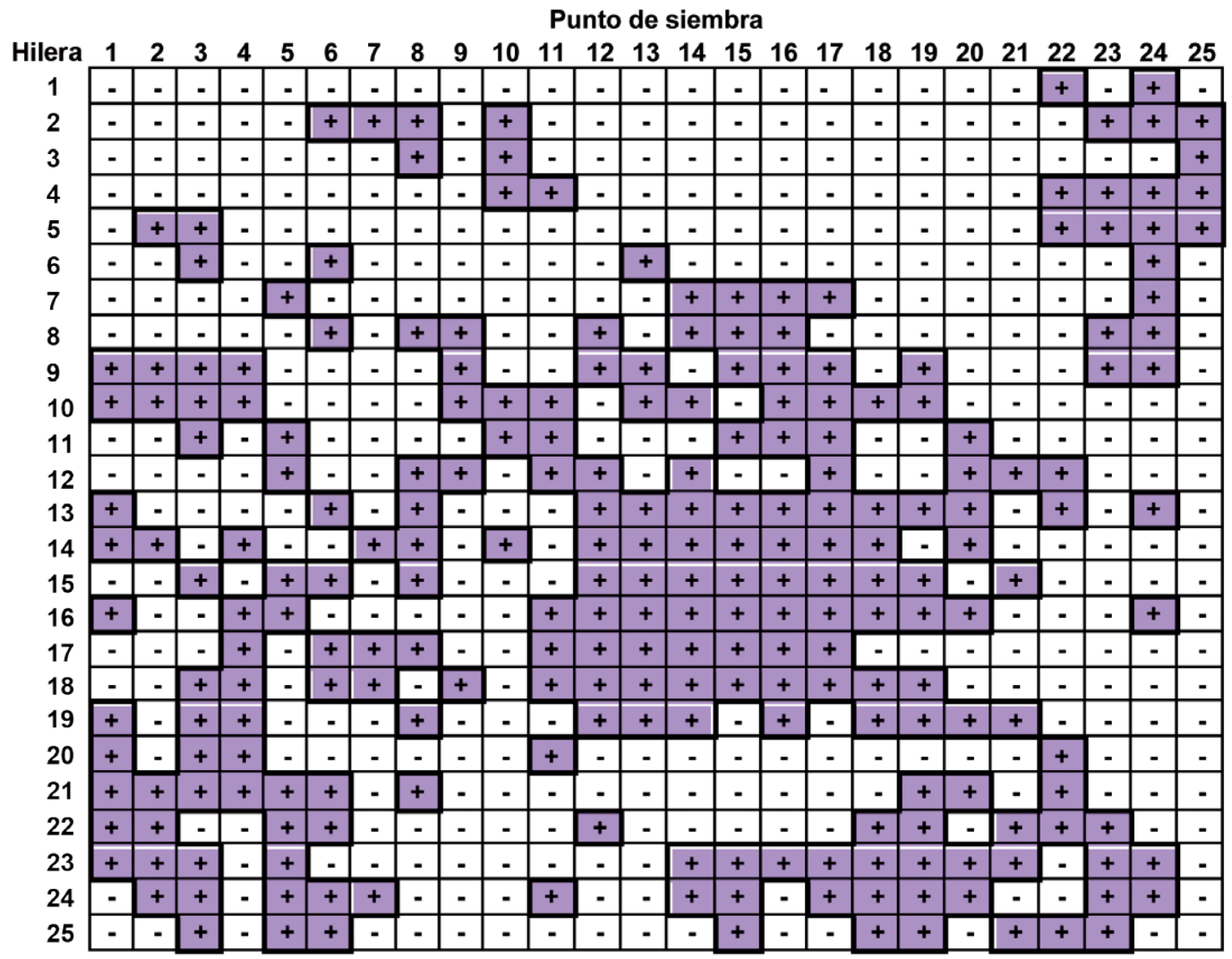

Figura 1. Patrón espacial del amachamiento (Aphelenchoides besseyi) en una siembra comercial de frijol de la variedad Cabécar, en un campo sembrado previamente con arroz. Puntos de siembra enfermos (+) y sanos (-). Concepción, Buenos Aires, Costa Rica. 2009. 


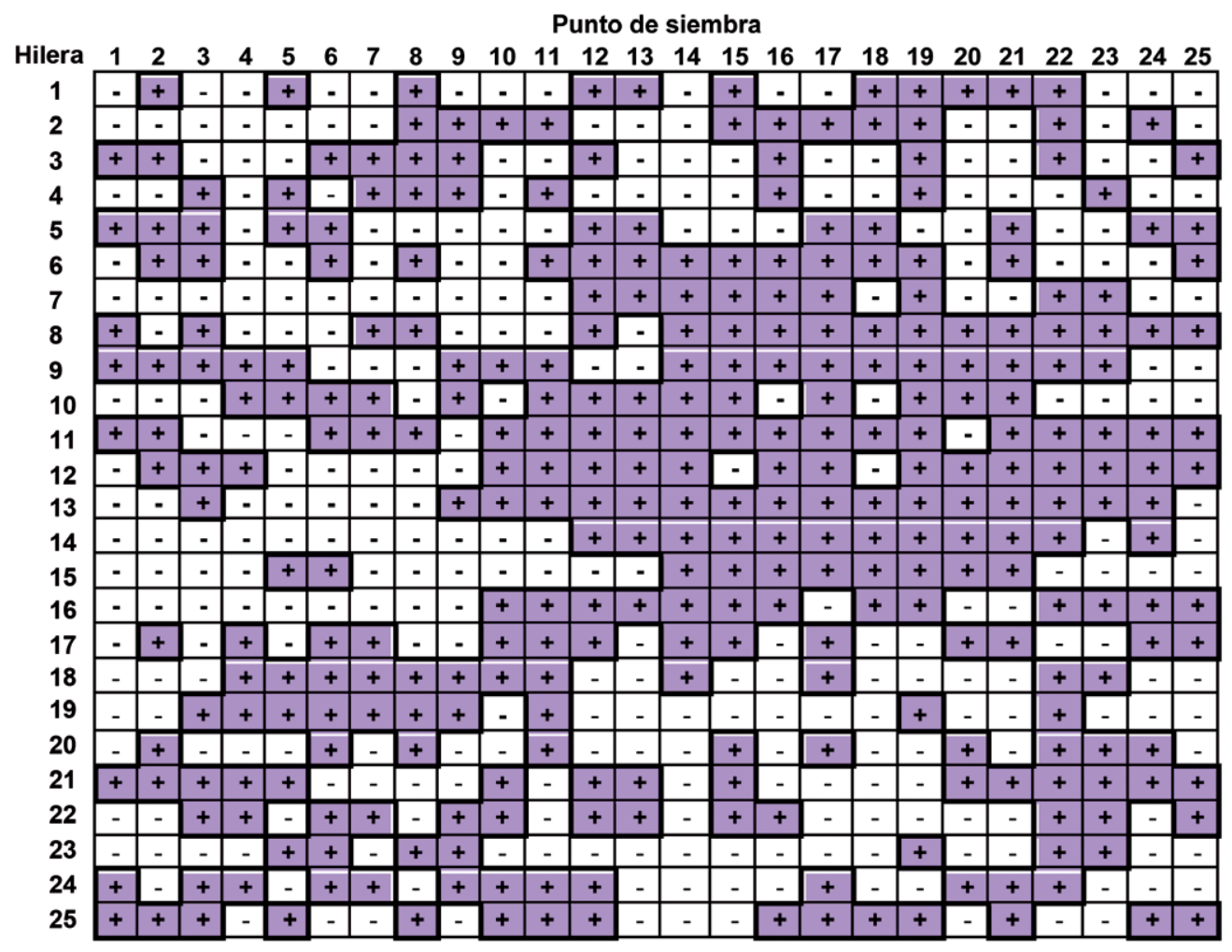

Figura 2. Patrón espacial del amachamiento (Aphelenchoides besseyi) en una siembra comercial de frijol de la variedad Cabécar, en un campo sembrado previamente con maíz. Puntos de siembra enfermos (+) y sanos (-). Veracruz, Pérez Zeledón, Costa Rica. 2009.

enfermedad (Figuras 1 y 2). Solo un bajo porcentaje de las hileras presentaron valores de $\mathrm{Z}$ menores a $-1,64$, indicativo de un patrón agregado dentro de la hilera (Campbell y Madden, 1990). De este modo, $36 \%$ de las hileras de la parcela en rotación con arroz (Cuadro 1) y 25,4\% de las de la parcela en rotación con maíz (Cuadro 2) presentaron un arreglo espacial agregado. Sin embargo, como lo indican Campbell y Madden (1990), esta metodología presenta algunos problemas para evaluar el patrón de enfermedades en parcelas grandes, ya que lo realiza únicamente dentro de las hileras y no en la parcela como un todo. Debido a lo anterior, se promedió el valor de $\mathrm{Z}$, y se obtuvo $-1,06$ y $-0,98$ para las parcelas sembradas previamente con arroz y maíz, respectivamente; lo que indica esa tendencia a la agregación observada en los mapas de campo (Figuras 1 y 2).
Además del análisis de corridas, se realizó un análisis de agregación por medio de los índices de varianza/media (V/M), Lloyd y Morisita para determinar el patrón espacial del amachamiento en plantaciones comerciales del cultivo. En ambas parcelas, con diferente rotación, se obtuvieron valores mayores a 1,0 para los tres índices (Cuadro 3), lo que confirma que la enfermedad efectivamente se presenta en forma agregada en condiciones de campo. Resultados similares se obtuvieron para otros nematodos fitoparásitos como Criconemella onoensis, Helicotylenchus erythrinae, Hemicriconemoides cocophillus y Pratylenchiis zeae en caña de azúcar en Martinica, donde se determinó el patrón agregado de estos mediante los índices de V/M y Morisita (Rossi et al., 1996). Igualmente, Siddiqui y Shaukat (2002) emplearon los índices de V/M, Lloyd y Morisita para determinar el patrón espacial 
Cuadro 1. Análisis de corridas para determinar el patrón espacial del amachamiento (Aphelenchoides besseyi) en una siembra comercial de frijol de la variedad Cabécar, establecida en un campo sembrado previamente con arroz. Concepción, Buenos Aires, Costa Rica. 2009.

\begin{tabular}{|c|c|c|c|c|c|c|}
\hline Hilera & $\mathbf{U}^{*}$ & $\mathbf{m}^{*}$ & $\mathbf{E}(\mathbf{U})^{*}$ & $\mathbf{s}(\mathbf{U})^{*}$ & $\mathbf{Z}^{*}$ & Conclusión \\
\hline 1 & 5 & 2 & 4,68 & 0,64 & 1,28 & Aleatorio \\
\hline 2 & 6 & 7 & 11,08 & 1,95 & $-2,35$ & Agregado \\
\hline 3 & 6 & 3 & 6,28 & 0,97 & 0,23 & Aleatorio \\
\hline 4 & 4 & 6 & 10,12 & 1,76 & $-3,20$ & Agregado \\
\hline 5 & 4 & 6 & 10,12 & 1,76 & $-3,20$ & Agregado \\
\hline 6 & 9 & 4 & 7,72 & 1,27 & 1,41 & Aleatorio \\
\hline 7 & 7 & 6 & 10,12 & 1,76 & $-1,49$ & Aleatorio \\
\hline 8 & 11 & 9 & 12,52 & 2,25 & $-0,45$ & Aleatorio \\
\hline 9 & 12 & 13 & 13,48 & 2,44 & $-0,40$ & Aleatorio \\
\hline 10 & 8 & 13 & 13,48 & 2,44 & $-2,04$ & Agregado \\
\hline 11 & 11 & 8 & 11,88 & 2,12 & $-0,18$ & Aleatorio \\
\hline 12 & 13 & 10 & 13,00 & 2,35 & 0,21 & Aleatorio \\
\hline 13 & 5 & 17 & 11,88 & 2,12 & $-3,01$ & Agregado \\
\hline 14 & 12 & 14 & 13,32 & 2,41 & $-0,34$ & Aleatorio \\
\hline 15 & 11 & 13 & 13,48 & 2,44 & $-0,81$ & Aleatorio \\
\hline 16 & 8 & 14 & 13,32 & 2,41 & $-2,00$ & Agregado \\
\hline 17 & 7 & 11 & 13,32 & 2,41 & $-2,41$ & Agregado \\
\hline 18 & 9 & 14 & 13,32 & 2,41 & $-1,58$ & Aleatorio \\
\hline 19 & 12 & 12 & 13,48 & 2,44 & $-0,40$ & Aleatorio \\
\hline 20 & 8 & 5 & 9,00 & 1,53 & $-0,33$ & Aleatorio \\
\hline 21 & 8 & 10 & 13,00 & 2,35 & $-1,92$ & Agregado \\
\hline 22 & 10 & 10 & 13,00 & 2,35 & $-1,07$ & Aleatorio \\
\hline 23 & 8 & 14 & 13,32 & 2,41 & $-2,00$ & Agregado \\
\hline 24 & 13 & 14 & 13,32 & 2,41 & 0,07 & Aleatorio \\
\hline 25 & 11 & 9 & 12,52 & 2,25 & $-0,45$ & Aleatorio \\
\hline \multicolumn{5}{|l|}{ Promedio } & $-1,06$ & \\
\hline \multicolumn{5}{|c|}{ Hileras con agregación } & & $36,0 \%$ \\
\hline
\end{tabular}

* U: número de corridas, m: número de plantas infectadas, $\mathrm{E}(\mathrm{U})$ : número esperado de corridas, s(U): desviación estándar del número de corridas y Z: valor crítico de distribución normal estándar.

de Meloidogyne javanica en berenjena, y Spiridonov et al. (2007) estudiaron la distribución espacial de los nematodos entomopatógenos Steinernema feltiae y $S$. affine en suelos dedicados a pasturas mediante el índice de Lloyd.
Los resultados obtenidos concuerdan con lo esperado para una enfermedad causada por un nematodo, cuya escasa movilidad le impide trasladarse a grandes distancias, lo que genera patrones espaciales típicamente agregados (Campbell y Noe, 1985; Noe y 
Cuadro 2. Análisis de corridas para determinar el patrón espacial del amachamiento (Aphelenchoides besseyi) en una siembra comercial de frijol de la variedad Cabécar, establecida en un campo sembrado previamente con maíz. Veracruz, Pérez Zeledón, Costa Rica. 2009.

\begin{tabular}{|c|c|c|c|c|c|c|}
\hline Hilera & $\mathbf{U}^{*}$ & m* & $\mathbf{E}(\mathbf{U})^{*}$ & $\mathbf{s}(\mathbf{U})^{*}$ & $\mathbf{Z}^{*}$ & Conclusión \\
\hline 1 & 13 & 11 & 13,32 & 2,41 & 0,07 & Aleatorio \\
\hline 2 & 9 & 11 & 13,32 & 2,41 & $-1,58$ & Aleatorio \\
\hline 3 & 13 & 11 & 13,32 & 2,41 & 0,07 & Aleatorio \\
\hline 4 & 15 & 9 & 12,52 & 2,25 & 1,33 & Aleatorio \\
\hline 5 & 11 & 12 & 13,48 & 2,44 & $-0,81$ & Aleatorio \\
\hline 6 & 12 & 15 & 13,00 & 2,35 & $-0,21$ & Aleatorio \\
\hline 7 & 7 & 9 & 12,52 & 2,25 & $-2,23$ & Agregado \\
\hline 8 & 9 & 17 & 11,88 & 2,12 & $-1,12$ & Aleatorio \\
\hline 9 & 6 & 18 & 11,08 & 1,95 & $-2,35$ & Agregado \\
\hline 10 & 11 & 14 & 13,32 & 2,41 & $-0,76$ & Aleatorio \\
\hline 11 & 7 & 20 & 9,00 & 1,53 & $-0,98$ & Aleatorio \\
\hline 12 & 8 & 17 & 11,88 & 2,12 & $-1,60$ & Aleatorio \\
\hline 13 & 5 & 17 & 11,88 & 2,12 & $-3,01$ & Agregado \\
\hline 14 & 5 & 12 & 13,48 & 2,44 & $-3,27$ & Agregado \\
\hline 15 & 5 & 10 & 13,00 & 2,35 & $-3,20$ & Agregado \\
\hline 16 & 6 & 13 & 13,48 & 2,44 & $-2,86$ & Agregado \\
\hline 17 & 16 & 14 & 13,32 & 2,41 & 1,32 & Aleatorio \\
\hline 18 & 9 & 12 & 13,48 & 2,44 & $-1,63$ & Aleatorio \\
\hline 19 & 9 & 10 & 13,00 & 2,35 & $-1,49$ & Aleatorio \\
\hline 20 & 17 & 10 & 13,00 & 2,35 & 1,92 & Aleatorio \\
\hline 21 & 9 & 15 & 13,00 & 2,35 & $-1,49$ & Aleatorio \\
\hline 22 & 14 & 13 & 13,48 & 2,44 & 0,42 & Aleatorio \\
\hline 23 & 9 & 7 & 11,08 & 1,95 & $-0,81$ & Aleatorio \\
\hline 24 & 12 & 13 & 13,48 & 2,44 & $-0,40$ & Aleatorio \\
\hline 25 & 13 & 15 & 13,00 & 2,35 & 0,21 & Aleatorio \\
\hline \multicolumn{5}{|l|}{ Promedio } & $-0,98$ & \\
\hline \multicolumn{6}{|c|}{ Hileras con agregación } & $25,4 \%$ \\
\hline
\end{tabular}

* U: número de corridas, m: número de plantas infectadas, $\mathrm{E}(\mathrm{U})$ : número esperado de corridas, $\mathrm{s}(\mathrm{U})$ : desviación estándar del número de corridas y Z: valor crítico de distribución normal estándar.

Cuadro 3. Índices de agregación para el amachamiento (Aphelenchoides besseyi) en parcelas comerciales de frijol de la variedad Cabécar provenientes de rotaciones con arroz y maíz. Veracruz de Pérez Zeledón y Concepción de Buenos Aires, Costa Rica. 2009.

\begin{tabular}{lccc}
\hline Parcela & \multicolumn{3}{c}{ Índice } \\
\cline { 2 - 4 } & V/M & Lloyd & Morisita \\
\hline Rotación arroz - frijol & 3,02 & 1,21 & 1,21 \\
Rotación maíz - frijol & 1,50 & 1,04 & 1,04 \\
\hline
\end{tabular}


Campbell, 1985; Arauz, 2011). Además, confirmaron lo encontrado por Barrantes (2006), quien determinó que el inóculo de $A$. besseyi se encuentra típicamente agregado. El patrón agregado del amachamiento es fácilmente observado en condiciones de campo, cuando el frijol llega al punto de cosecha y las plantas sanas pierden el follaje, ya que las enfermas permanecen verdes y con follaje (Figura 3), condición que se presenta debido a que la planta como consecuencia de la infestación no produce vainas (sumideros) o tiene muy pocas, lo que le permite utilizar los fotoasimilados producidos para continuar con su crecimiento vegetativo y mantener su coloración.

A la fecha, por lo reciente del diagnóstico correcto de la enfermedad (Chaves, 2011; Chaves et al., 2013), no se cuentan con estudios sobre medidas para el manejo del patógeno en el cultivo de frijol. El combate químico de nematodos del género Aphelenchoides en otros cultivos se ha llevado a cabo mediante el uso de insecticidas como diazinón y abamectina, y nematicidas como oxamil, que logran reducir las poblaciones pero no lo erradican completamente ( $\mathrm{La}$ Mondia, 1999; Jagdale y Grewal, 2002). Este tipo de agroquímicos acarrea una alta toxicidad, riesgo para la salud humana y graves daños ambientales (Jagdale y Grewal, 2004; Van der Putten et al., 2006), por lo que al conocer que la enfermedad efectivamente se presenta en forma agregada, su combate químico se puede llevar a cabo en forma dirigida y en etapas tempranas del cultivo. De esta forma el combate de la enfermedad se puede realizar cuando inician los primeros parches y así evitar la expansión del problema en la plantación. Esto implica menor cantidad de producto a aplicar, menor daño ambiental, menor tiempo de exposición del aplicador y menor costo económico.

Al comparar entre las parcelas establecidas en relación con el cultivo anterior, en la rotación con arroz se visualizó mejor el patrón agregado y presentó índices de agregación más elevados (Cuadro 3), en concordancia con los valores más bajos obtenidos en el análisis corridas (Cuadro 1). Esto se debe básicamente a la redistribución del inóculo que ocurre en un

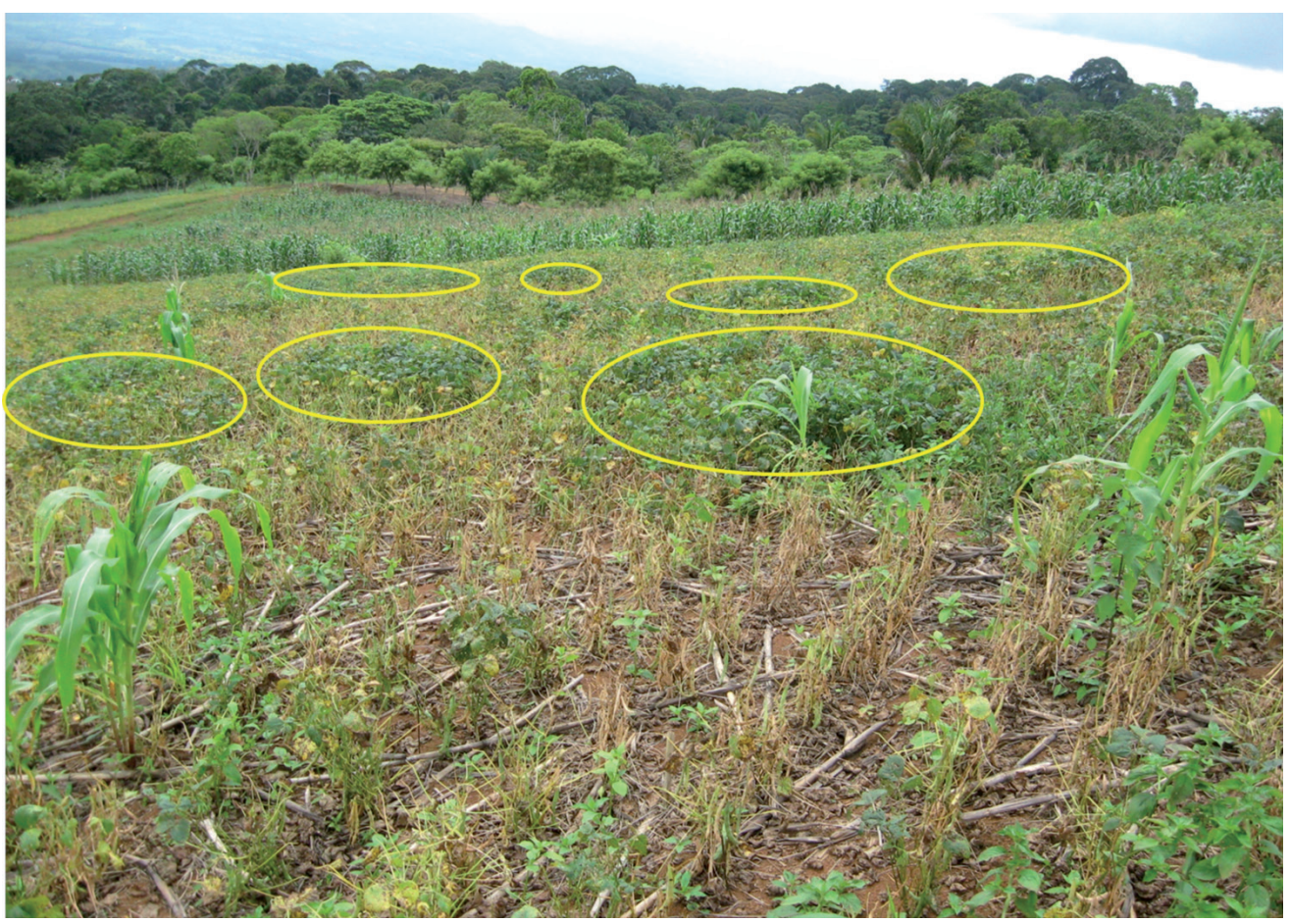

Figura 3. Patrón espacial del amachamiento en una siembra comercial de frijol en el momento de cosecha. Pueblo Nuevo, Buenos Aires, Costa Rica. 2009. 
terreno previamente sembrado con maíz - hospedante alterno de A. besseyi - (Barrantes, 2006; Arauz, 2011), ya que el productor esparce las cañas de maíz por toda la parcela antes de la siembra de frijol. Al respecto, Campbell y Noe (1985) mencionan que el análisis del patrón espacial de un patógeno de suelo, proporciona información de la influencia de las prácticas culturales realizadas en el campo de cultivo, como en este caso.

Debido a la redistribución del inóculo hecha por el productor y a que probablemente la población de $A$. besseyi sea abundante por la rotación con cultivos hospederos como arroz y maíz (Chaves y Araya, 2012a), es que se enmascara el patrón agregado del nematodo en campo y no se visualiza claramente su agregación. No obstante, se demostró por medio de los índices de varianza/media, Lloyd y Morisita que el amachamiento presenta un patrón agregado en los terrenos de cultivo.

\section{AGRADECIMIENTOS}

A la Fundación CRUSA (convenio CRUSA-CSIC) y a la Universidad de Costa Rica (Proyecto 736-A4141 inscrito en Vicerrectoría de Investigación), instituciones que financiaron el desarrollo de esta investigación. A los señores Adalberto Morera y Marvin Mora, quienes brindaron su colaboración y cedieron sus plantaciones comerciales de frijol para la realización de este trabajo.

\section{LITERATURA CITADA}

Arauz, L. 2011. Fitopatología: un enfoque agroecológico. 2 ed. Editorial de la Universidad de Costa Rica, San José, Costa Rica.

Araya, C. 2001. Distribución agroecológica de enfermedades del frijol en Costa Rica. En: J. Hernández, y R. Araya, editores, V Taller Anual de Resultados de Investigación y Transferencia en Tecnología del PITTA-Frijol. 8 al 9 de agosto, 2001. Editorial de la Universidad de Costa Rica, Alajuela, Costa Rica. p. 111-117.

Araya, C., y J. Hernández. 2003. Distribución agroecológica de enfermedades de frijol en Costa Rica. Manejo Integrado de Plagas y Agroecología 68:26-33.

Araya, C., y J. Hernández. 2006. Guía para la identificación de las enfermedades del frijol más comunes en Costa Rica. Ministerio de Agricultura y Ganadería (MAG), San José, Costa Rica.
Araya,C.2008.Enfermedades y su combate.En: J.Hernández, y L. Ramírez, editores, Cultivo de frijol (Phaseolus vulgaris). Manual de recomendaciones técnicas cultivo de frijol. Instituto Nacional de Innovación y Transferencia en Tecnología Agropecuaria (INTA), San José, Costa Rica. p. 63-72.

Barrantes, W. 2006. Epidemiología de la falsa mancha angular (Aphelenchoides spp.) en el cultivo de frijol (Phaseolus vulgaris L.). Tesis M.Sc., Universidad de Costa Rica, San José, Costa Rica.

Campbell, C., y J. Noe. 1985. The spatial analysis of soilborne pathogens and root diseases. Annu. Rev. Phytopathol. 23:129-148

Campbell, C., y L. Madden. 1990. Introduction to plant disease epidemiology. Wiley, New York, USA.

Chaves, N. 2011. Epidemiología del amachamiento y cuantificación de las pérdidas que ocasiona en el cultivo de frijol (Phaseolus vulgaris L.). Tesis M.Sc., Universidad de Costa Rica, San José, Costa Rica.

Chaves, N., y C. Araya. 2012a. Efecto de la rotación de cultivos en la incidencia del amachamiento (Aphelenchoides besseyi Christie) en frijol. Agronomía Costarricense 36(2):61-70.

Chaves, N., y C. Araya. 2012b. Pérdidas causadas por el amachamiento del frijol (Aphelenchoides besseyi Christie) y reacción del germoplasma comercial al patógeno. Agron. Mesoam. 23:1-12.

Chaves, N., E. Cervantes, I. Zabalgogeazcoa, y C. Araya. 2013. Aphelenchoides besseyi Christie (Nematoda: Aphelenchoididae), agente causal del amachamiento del frijol común. Tropical Plant Pathology 38(3):243-252.

Chitwood, D. 2002. Phytochemical based strategies for nematode control. Annu. Rev. Phytopathol. 40:221249.

Christie, J. 1982. Nematodos de los vegetales: su ecología y control. Editorial Limusa, México.

Fortuner, R., y K. Orton. 1975. Review of the literature on Aphelenchoides besseyi Christie, 1942, the nematode causing "white tip" disease in rice. Helminthological Abstracts 44(1):1-40.

Jagdale, G.B., y P.S. Grewal. 2002. Identification of alternatives for the management of foliar nematodes in floriculture. Pest Management Science 58(5):451-458.

Jagdale, G.B., y P.S. Grewal. 2004. Effectiveness of a hot water drench for the control of foliar nematodes Aphelenchoides fragariae in floriculture. J. Nematol. 36(1):49-53.

La Mondia, J.A. 1999. Efficacy of insecticides for control of Aphelenchoides fragariae and Ditylenchus dipsaci in 
flowering perennial ornamentals. Supplement to the J. Nematol. 31(4S):644-649.

Noe, J., y C. Campbell. 1985. Spatial pattern analysis of plant parasitic nematodos. J. Nematol. 17:86-93.

Rodríguez, S., y X. Fernández. 2008. Consumo de frijoles en familias costarricenses y relación con condición geográfica y características de la persona encargada de la preparación. Avances en Seguridad Alimentaria y Nutricional (Costa Rica) Año 2008:9-14.

Rossi, J.P., L. Delaville, y P. Quénéhervé. 1996. Microspatial structure of a plant-parasitic nematode community in a sugarcane field in Martinique. Appl. Soil Ecol. 3:17-26.

Salas,L., y E. Vargas. 1984.El nematodofoliar Aphelenchoides besseyi Christie (Nematodo: Aphelenchoididae) como causante de la falsa mancha angular del frijol en Costa Rica. Agronomía Costarricense 8:65-68.

Salazar, J. 2002. La actividad de frijol en Costa Rica en el ciclo 2001-2002. En: R. Araya, editor, VI Taller Anual de Resultados de Investigación y Transferencia en Tecnología del PITTA-FRIJOL. 21 al 22 de agosto, 2002. SIEDIN, San José, Costa Rica. p. 7-15.
Salazar, J. 2003. Perfil de la actividad de frijol en Costa Rica. En: R. Araya, C. Araya, J. Hernández, editores, VII Taller Anual de Resultados de Investigación y Transferencia en Tecnología del PITTA-FRIJOL. 2 al 4 de setiembre, 2003. Santo Domingo, Heredia, Costa Rica. p. 1-6.

Siddiqui, I.A., y S.S Shaukat. 2002. Spatial pattern analysis of root rot-root knot disease complex in an infested egg plant field. Nematología Mediterránea 30:131135.

Spiridonov, S.E., M. Moens, y M.J. Wilson. 2007. Fine scale spatial distributions of two entomopathogenic nematodes in a grassland soil. Appl. Soil Ecol. 37(3):192-201.

Van der Putten, W.H., R. Cook, S. Costa, K.G. Davies, M. Fargette, H. Freitas, W.H.G. Hol, B.R. Kerry, N. Maher, T. Mateille, M. Moens, E. De la Peña, A. Piśkiewicz, A. Raeymaekers, S. Rodríguez, y A.W.G. Van der Wurff. 2006. Nematode interactions in nature: models for sustainable control of nematode pests of crop plants? Adv. Agron. 89:227-260. 